\title{
Clinical Skills Verification: a Problematic Examination
}

\author{
Richard Balon ${ }^{1}$ (D) Eugene V. Beresin ${ }^{2} \cdot$ Anthony P. S. Guerrero ${ }^{3} \cdot$ Mary K. Morreale $^{1} \cdot$ Rashi Aggarwal $^{4}$. \\ Alan K. Louie ${ }^{5}$. Adam M. Brenner ${ }^{6}$
}

Received: 2 December 2019 / Accepted: 4 December 2019 / Published online: 19 December 2019

(C) Academic Psychiatry 2019

Good interviewing and careful listening are the most important parts of a psychiatrist's skill set. Psychiatry residents are trained in interviewing via observation of their teachers interviewing patients with differing clinical profiles in multiple settings, by being observed interviewing patients and receiving feedback about their interviewing skills, and through reading various texts on psychiatric interviewing. Similarly, the ability for the resident to listen effectively is an important component of all clinical interviewing, and residents are taught to listen through similar processes.

The evaluation of residents' interviewing skills is an important part of their overall competency evaluation. Training programs historically conducted this evaluation in several ways: observed interviews during various rotations; conducting clinical skills evaluations (CSEs) based on the competency requirements of the Accreditation Council for Graduate Medical Education (ACGME) and the American Board of Medical Specialties (ABMS) multiple times a year; and an annual clinical examination, which was previously known as the mock board examination and modeled after the former Part II (oral examination) of the certification examination of the American Board of Psychiatry and Neurology, Inc. (ABPN). Patient interviews are also components of several Milestones, including patient care 1: psychiatric evaluation; patient care 2: psychiatric formulation and differential diagnosis; and patient care 3: treatment planning and management. Finally, the examination of

Richard Balon

rbalon@wayne.edu

Wayne State University, Detroit, MI, USA

2 Harvard Medical School, Boston, MA, USA

3 University of Hawai'i John A. Burns School of Medicine, Honolulu, HI, USA

4 Rutgers New Jersey Medical School, Newark, NJ, USA

5 Stanford University, Stanford, CA, USA

6 University of Texas Southwestern Medical Center, Dallas, TX, USA interviewing skills, formulation, and treatment plan was also part of the ABPN certification process.

\section{Historical Background}

The examination of interviewing skills was part of the certification process by the ABPN since its inception. The ABPN was founded in 1934, and the first examination was held in 1935. The oral examination was part of the certification process in various forms for the following 73 years, until 2008. From 1935 to 1946 , both psychiatry and neurology took the same examination ( $3 \mathrm{~h}$ psychiatry, $3 \mathrm{~h}$ neurology), then two separate examinations were developed [1], and oral examination continued to be a main part of the psychiatry certification examination. The ABPN certification examination was later composed of two parts: Part I, a cognitive examination focused on knowledge of psychiatry and neurology (first, a paper test taken by pencil; later, a test administered by computer), and Part II, patient section, an oral examination focused on patient evaluation.

Since about 1976, Part II of the examination consisted of two sections: patient $(1 \mathrm{~h})$ and audiovisual $(1 \mathrm{~h})$. The patient section was a 30 -min interview of a real patient (previously unknown to the candidate) followed by a 30min discussion. In the discussion phase, the candidate was asked to summarize the case and present a formal psychiatric, medical, family, and social history; mental status examination; formulation; differential diagnosis; prognosis estimate; and management in a biopsychosocial framework, and was then questioned about the case by two observing ABPN examiners (plus a short observation by a rotating senior examiner). The audiovisual section consisted of watching a 25-30-min video followed by a 30-min session similar to the patient section discussion with two ABPN examiners (and again, short observation by a rotating senior examiner). In 2006, the ABPN replaced the audiovisual section with a 1-h vignette section composed of discussion of three brief written vignettes and one video vignette. 
Candidates and examiners from different parts of the USA and, at times, Canada would assemble at selected sites several times per year for the Part II examination. In an effort to reduce the possibility of bias and conflict of interest, the examiners were completely blind to the candidate's status. They knew only the candidate's name and not the candidate's address or training program or whether the candidate was a repeat examinee. If either the candidate or examiner knew each other, reassignments were required to either different examiners or a different examining team.

In 2008, the ABPN decided to replace Part II of its examination with clinical skills verification (CSV) examination administered by residency training programs. Part I, the cognitive, computer-administered examination that focused on psychiatric knowledge became the certification examination. ABPN leadership concluded about the oral examinations that "significant enhancements in their validity could not be achieved given resource constraints, and the financial and logistical problems associated with them were increasing" ([2], p. 98). The ABPN president and chief executive officer also wrote, "The ABPN well understands that certain competencies can only be assessed by direct observation. It is also aware, however, that identifying serious deficiencies in basic interviewing and case presentation skills in an oral examination after candidates have already finished their residencies makes remediation of those skills extremely difficult" ([3], p. 4399). The interviewing skills examination/certification then became the responsibility of training programs and an unfunded mandate.

\section{CSV Examination}

The ABPN requires that residents successfully complete three CSV examinations during their training, with documentation of their completion provided by their program director via the ABPN website [4]. Documentation must include confirmation that the candidate successfully completed the three examinations (on different patients) and the names of all three examiners and dates of the examinations. The examiners must be ABPN certified, and at least two examinations must be done by different evaluators. The three successful CSV examinations are required to be documented by July 31 of the year the candidate takes the ABPN certification examination.

The CSV requirements are similar to the former ABPN Part II live patient. The three components to be formally considered in the evaluation are physician-patient relationship, psychiatric interview including mental status examination, and case presentation. The evaluator judges performance on all of these three components, and the resident must have acceptable performance on each in order to pass. Selection of the patient is at the discretion of the program director or the program. Patients must be unknown to the examinee. Patients who do not speak English could be used only if the examinee and examiner fluently speak the patient's language; translators are not permissible. At a minimum, each CSV examination should last $45 \mathrm{~min}$, with a minimum of $30 \mathrm{~min}$ given to the candidate to conduct the interview and a minimum of 10 15 min to present the case. The CSV examination can last longer if need be. The evaluator can give feedback to the examinee, if appropriate. The evaluation must be completed on one of two ABPN-approved forms available on the ABPN website (Psych CSV v1 and Psych CSV v2) [4] or a form proposed by the program and approved by the ABPN, and programs may use additional evaluation tools. Residents can take the CSV examination any time during their training, but the examinations should be spread apart. With relief from time pressure, residents can take the CSV examination multiple times if necessary, without affecting their admissibility to the ABPN certification examination, because only the three successfully completed CSVs are reported to the ABPN. Remediation of any failed CSV examination is up to the training program (e.g., requiring an increased number of observed interviews). CSVs completed during residency are valid for 7 years following completion of training. The CSVs completed outside of residency training (e.g., for physicians who trained before the full adoption of CSVs in lieu of the Part II exam) are valid for 7 years following the completion of the last CSV examination, which has to be completed within 5 years of the first CSV examination.

Candidates pursuing ABPN certification in child and adolescent psychiatry must pass three similarly formatted CSV examinations on youth representing at least two of the following age groups: preschool, school age, and adolescent. One of the child and adolescent CSVs may count toward the three CSVs required for ABPN certification in general psychiatry. Full details of the CSV requirements can be found on the ABPN website [4].

ACGME-accredited programs (and thus indirectly the ACGME), according to the residency review committee guidelines [5], are required to certify the competency of individuals. Program directors are required to verify that the resident has demonstrated the knowledge, skills, and behaviors necessary to graduate and enter autonomous practice. It could be argued that the ACGME has taken the place of the ABPN in requiring standardized certification of individuals and programs and that the ABPN is potentially becoming nonessential in certifying clinical competency.

\section{Is the CSV Examination Valid, Reliable, Objective, and Free of Conflict of Interest?}

After changes are instituted, we feel it is a good approach to look back at measures and processes after some time to see whether expectations were met. In our experience, however, 
such an approach is uncommon in medical education, where processes are frequently adopted without a subsequent careful look at their consequences (with exceptions, such as evaluations of the consequences of changes to duty hours). Because the CSV examination has been around for over a decade, we think it is time to examine critically the issues of the CSV'S validity, reliability, objectivity, and absence of conflicts of interest. We have been able to find only four articles addressing CSV examinations in psychiatry since its inception [2, 6-8].

Juul and colleagues [2] electronically surveyed all neurology, child neurology, and psychiatry program directors, and a convenience sample of graduates who applied for the 2012 ABPN certification examination and assessed their experience with the CSV (labeled CSE). Most program directors and residents reported that residents received verbal feedback and that the CSE "definitely" or "somewhat" accurately measured their relationship with patients, interviewing skills, and case presentation skills. Interestingly, $70 \%$ of responding program directors reported that the most common activities assigned on the basis of CSE performances were additional CSEs and patient interviews observed by faculty, while $87 \%$ of responding residents reported no post-CSE assigned activity. The study was hampered by low response rates of $29 \%$ for program directors and $53 \%$ for residents. The article by Jibson and colleagues [6] summarized the recommendation of the ABPN Task Force on Clinical Skills Verification Rater Training, concluding, "The implementation of the CSV process provides psychiatry training programs with an excellent opportunity to assess how interviewing skills are taught and evaluated" (p. 363). Rao and colleagues [7] explored resident experience with the CSV process and differences between resident physicians who were US medical graduates (USMG) and residents who were international medical graduates (IMG). The majority of residents, both USMG and IMG, felt that the CSV was helpful in improving their interviewing skills. The IMG residents were more likely to experience feedback as excessively negative and critical and wanted more supervised interviews.

\section{Validity}

One could argue that because the former ABPN Part II oral examination was considered a valid evaluation of interviewing skills, the CSV also could be considered valid. The validity of the Part II live interview, however, was probably enhanced by the presence of two experienced examiners together with a brief presence of a senior examiner and by preparatory discussions and rules enforced by the ABPN. Furthermore, training was also instituted as to what might constitute a passing performance. In contrast, there is no national requirement or observational model for training CSV examiners. The means by which examiners conduct their assessments is therefore likely to vary widely and perhaps arbitrarily.

It also could be argued that face validity of the CSV may be superior to the Part II oral examination in a manner that is analogous to the benefits of effectiveness (instead of efficacy) of clinical trials. The CSV could be happening in actual clinical settings, even in the context of actual workflow, and thus would be closer to clinical reality than seeing a paid patient in the context of the ABPN Part II oral examination. It is not clear, however, whether that is the manner in which the examination happens in residency training programs, and no official guidance to this process is available.

One general criticism of the CSV has been its failure to include a diagnosis, differential diagnosis, and treatment plan in consideration of whether the candidate meets passing standards, although these components were mandatory parts of the annual mock oral examination of training programs and of Part II of the ABPN certification examination. When the CSVs were first proposed and the benchmarks were defined and disseminated by the ABPN, many involved in residency training objected to including simply the physician-patient relationship, the psychiatric interview with a mental status examination, and the case presentation. It was argued that psychiatry residents should demonstrate a sophistication and competence in an understanding and management of a patient's history and concerns by reporting a provisional diagnosis/differential diagnosis, treatment plan, and prognosis. Some programs included these sections of an annual clinical examination in their CSVs. Additionally, some programs counted these CSVs toward the ACGME's requirement for formal CSEs, which must be administered annually to all residents and include all the elements of the CSV plus formulation, differential diagnosis, and provisional treatment plan.

\section{Reliability}

Dalack and Jibson [8] described the experience of implementing the CSV examination in their program. They embedded the CSV experience into clinical rotations through the 4 years of residency training and aimed to complete 6-12 evaluations for each resident each year. They argued that 8-12 clinical assessments would provide an optimal estimate of resident performance, with tight confidence intervals around the average score, on the basis of work by Norcini et al. [9]. However, Norcini et al. used a much shorter internal medicine examination/encounter (mean time $15 \mathrm{~min}$ ) with $5 \mathrm{~min}$ of feedback, and the internal medicine residents were evaluated on 1-8 evaluations [9]. This study also had several limitations. Thus, it is not clear whether these numbers could be transferred reliably into psychiatry's experience with the CSVs.

It is possible that conducting a higher number of CSVs may increase reliability if programs conduct sessions comparing these examinations or conduct a number of examinations 
being watched by several evaluators with a follow-up discussion including comparisons of ratings. Nevertheless, it is not clear whether programs are checking for reliability or whether all programs have the available personnel to conduct multiple exercises.

\section{Objectivity}

The objectivity of the CSV examination is debatable, and no good data addressing the issue are available. It seems that consideration of objectivity (and conflict of interest) was an important part of the ABPN Part II oral examination. As noted earlier, examiners were blinded to the candidate's information and status. We believe that personal knowledge of the resident by the examining faculty during the CSV examination may have a problematic effect on objectivity in creating both positive and negative halo effects and that faculty discussions among themselves outside the CSV examination also can contribute to lack of objectivity.

\section{Conflict of Interest}

The residency program manages the CSV examination, and the residency program is invested in the success of its trainees, both practically and psychologically. The ACGME, graduate medical education offices, and prospective applicants use board passing rates to evaluate residencies. A residency program that does not see the vast majority of its residents successfully pass through the hurdle of CSV examinations is likely to experience repercussions in recruiting future residents. At the same time, given that residency programs are understandably proud of their ability to teach competent practice of psychiatry, it is potentially psychologically difficult for a program to conclude that it is unable to help a resident progress. We would add that a workforce issue potentially creates a conflict of interest for program directors and examiners when services and programs are harmed by failing residents.

Dalack and Jibson [8] addressed the possibility of conflict of interest among training directors, acknowledging that program directors "may be motivated to achieve a high pass-rate as a reflection of program quality or to elevate resident morale. This is particularly true if CSV is seen primarily as a summative evaluation, or merely as an obstacle to ABPN certification" (p. 124). They stated, "The use of CSV early and often as a source of formative feedback helps address this issue" and that "many struggling residents, with sufficient instruction and feedback, will reach an appropriate level of performance for graduation," whereas other residents who will not reach this level will transition out of training before graduation ([8], $\mathrm{p}$. 124). Whether this outcome really happens and whether the conflict of interest of program directors is somehow resolved by this process remains to be studied. The passing rate of the ABPN examination between 2014 and 2018 was $89 \%$, an improvement over the passing rates during the Part II era. Considering that a substantial number of residents failed Part II, we should be seeing at least some residents leaving residency training for poor CSV performance. Are they? We do not know and currently lack the data to assess. One of the possible explanations is the conflict of interest of program directors and training programs. We need to research whether this is a possibility. Without data demonstrating otherwise, we view conflict of interest as an inherent feature of the CSV examination.

\section{Conclusion}

The end of patient-based oral certification examination was "vigorously challenged because of the critical nature of the skills that were evaluated. Program directors were concerned that the new requirements represented an unfunded mandate and that faculty members would find it difficult to serve as both coaches and judges" ([2], p. 100). Nevertheless, the ABPN mandated the use of CSV examination as a certifying examination without researching questions raised here and considering available personnel issues (which could be considerable, as illustrated above), and the field acquiesced. While some of the arguments for ending the Part II of the ABPN examination may be understandable, the issue of financial constraints should not have been a factor. The ABPN should have been able to examine issues raised here (validity, reliability, objectivity, conflict of interest, lack of examiner training) and develop different models of oral examinationwith examiner training included - or continue the previous model, capitalizing on substantial, and growing assets [10, 11]. The argument could be made, as some of us have stated before [10], that the role of the ABPN in the process of certification is a bit unclear considering the fact that the ABPN certification examination is yet another cognitive (i.e., medical knowledge) examination after four cognitive examinations during general psychiatry training and two in child and adolescent psychiatry fellowship, and the evaluation of clinical competence/skills is left to the training programs accredited by the ACGME. Thus, we believe that the process of evaluating clinical skills evaluation and certifying resident physicians should be reexamined and made more objective, free of conflicts of interest, and its validity should be increased. We also feel that the ABPN should reclaim its traditional role in certifying clinical skills in addition to its current role.

Alternatives that could be considered include restoring the former model of ABPN oral examination; using simulation and creating simulation centers for examinations; using remote access methods such as used for telepsychiatry in the CSV process so that residents and examiners are from different departments and unknown to each other; organizing mock board-style (e.g., with two examiners) examinations among 
geographically close programs, with exchanges of examiners; arranging for vetted and trained examiners to come to programs from outside to examine graduating residents on various types of patients in different settings arranged by the programs; creating a national center(s) akin to the United States Medical Licensing Examination (USMLE) Part II clinical skills examination center; or a combination of these options. Proper training for examiners in all these options would need to be exercised. A new form for evaluation of the oral patient examination that would include differential diagnosis, diagnosis, and management plan should be created and vetted, and its validity and reliability need to be studied.

Interviewing, listening, establishing an effective psychiatrist-patient relationship, summarizing the case, and outlining its management are our field's core skills. The evaluation of these skills is enormously important. We need uniform, national standards for certification of clinical skills. The certification process using the CSVs administered by training programs is problematic and subject to conflict of interest. Overwhelmed by many other issues, program directors, and programs acquiesced to it. It is time to reevaluate this examination and process of certification, perform rigorous studies of the process and its outcomes, and consider creating a new, more comprehensive (including differential diagnosis, diagnosis, formulation, prognosis, and management) certification examination, with some degree of validity and objectivity, and free of conflict of interest.

\section{Compliance with Ethical Standards}

Disclosure On behalf of all authors, the corresponding author states that there is no conflict of interest.

\section{References}

1. Koehler PJ. The four horsemen and the American Board of Psychiatry and Neurology (ABPN). J Hist Neurosci. 2018;27: 292-302.

2. Juul D, Brooks BA, Jozefowicz R, Jibson M, Faulkner L. Clinical skills assessment: the effects of moving certification requirements into neurology, child neurology, and psychiatry residency training. J Grad Med Educ. 2015;7:98-100.

3. Faulkner LR. Graduate psychiatric education. In: Sadock BJ, Sadock VA, Ruiz P, editors. Kaplan \& Sadock's Comprehensive Textbook of Psychiatry. Ninth ed. Philadelphia: Lippincott Williams \& Wilkins; 2009. p. 4396-410.

4. American Board of Psychiatry and Neurology. Available at https:// www.abpn.com/. Last accessed 26 November 2019.

5. Accreditation Council for Graduate Medical Education. Available at https://www.acgme.org/. Last accessed 26 November 2019.

6. Jibson MD, Broquet KE, Anzia JM, Beresin EV, Hunt JI, Kaye D, et al. Clinical skills verification in general psychiatry: recommendations of the ABPN Task Force on rater training. Acad Psychiatry. 2012;36:363-8.

7. Rao NR, Kodali R, Mian A, Ramtekkar U, Kamarajan C, Jibson MD. Psychiatric residents' attitudes toward and experiences with the clinical-skills verification process: a pilot study on U.S. and international medical graduates. Acad Psychiatry. 2012;36:316-22.

8. Dalack GW, Jibson MD. Clinical skills verification, formative feedback, and psychiatry residency trainees. Acad Psychiatry. 2012;36: 122-5.

9. Norcini JJ, Blank LL, Duffy FD, Fortna GS. The mini-CEX: a method for assessing clinical skills. Ann Intern Med. 2003;138: 476-81.

10. Balon R, Beresin EV, Guerrero A. Medical-education-industrial complex? Acad Psychiatry. 2018;42:495-7.

11. Drolet BC, Tandon VJ. Fees for certification and finances of medical specialty boards. JAMA. 2017;318:477-9.

Publisher's Note Springer Nature remains neutral with regard to jurisdictional claims in published maps and institutional affiliations. 